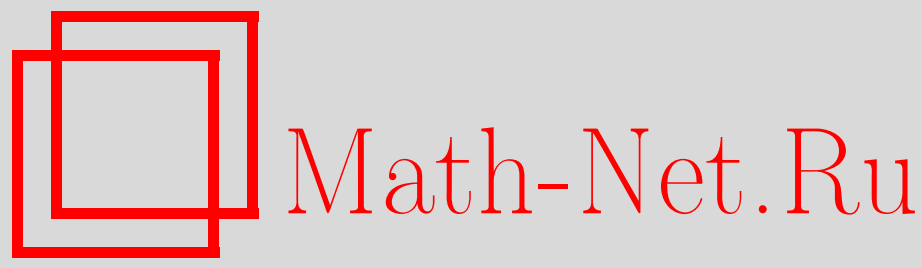

Л. А. Калякин, Синхронизация в неизохронной неавтономной системе, ТМФ, 2014, том 181, номер 2, 243-253

DOI: https://doi.org/10.4213/tmf8720

Использование Общероссийского математического портала Math-Net.Ru подразумевает, что вы прочитали и согласны с пользовательским соглашением http://www . mathnet.ru/rus/agreement

Параметры загрузки :

IP : 54.164 .48 .24

26 апреля 2023 г., 18:23:03

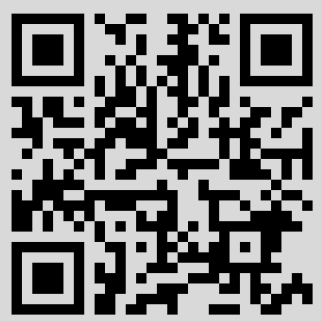




\title{
ФИЗИКА
}

Том 181, № 2

ноябрь, 2014

(C) 2014 г. Л. А. Калякин*

\section{СИНХРОНИЗАЦИЯ В НЕИЗОХРОННОЙ НЕАВТОНОМНОЙ СИСТЕМЕ}

\begin{abstract}
Исследована модельная система неавтономных нелинейных дифференциальных уравнений, возникающая в теории магнитодинамики. Найдены ограничения на параметры, при которых существуют решения со стабилизирующейся фазой, устойчивые по Ляпунову. Такие решения описывают явление синхронизации в неизохронной системе с медленно меняющимися параметрами.
\end{abstract}

Ключевые слова: нелинейные колебания, асимптотика, синхронизация, устойчивость.

DOI: $10.4213 / \operatorname{tmf} 8720$

\section{1. ВВЕДЕНИЕ}

Объектом исследования является модельная система уравнений

$$
\frac{d \rho}{d t}=-\gamma \rho+\left(a t+a_{0}\right) \cos \psi, \quad \frac{d \psi}{d t}=\rho+\delta t+\delta_{0}-\left(b t+b_{0}\right) \sin \psi,
$$

которая рассматривается при различных значениях параметров $a, b, \delta, \gamma$. Константы $a_{0}, b_{0}, \delta_{0}$ не играют особой роли и приводятся здесь для общности. Ввиду инвариантности структуры уравнений относительно преобразований $\psi \mapsto \psi+\pi$, $b \mapsto-b$ и $\rho \mapsto-\rho, \psi \mapsto-\psi, \delta \mapsto-\delta$ можно считать $b, \delta>0$. Константа $\gamma$ считается положительной, поскольку в задачах физики соответствует коэффициенту диссипации.

Цель работы состоит в выявлении ограничений на параметры, при которых существует устойчивое решение с компонентой $\psi(t)$, стабилизирующейся на бесконечности (при $t \rightarrow \infty$ ). Такие решения ассоциируются с явлением синхронизации [1].

Легко понять, что из-за растущих коэффициентов вторая компонента $\rho(t)$ в таких решениях обязана неограниченно расти при $t \rightarrow \infty$. Подобная ситуация в нелинейных осциллирующих процессах связывается с явлением авторезонанса [2]; в этом направлении похожая система анализировалась в работе [3]. Однако результаты об устойчивости для систем с диссипацией радикально отличаются от тех, что известны для моделей авторезонанса в отсутствии диссипации [4]. Как мы увидим далее,

* Институт математики с Вычислительным центром РАН, Уфа, Россия.

E-mail: klenru@mail.ru 
авторезонансные решения с растущей компонентой $\rho(t)$, которые удается построить, обладают экспоненциальной устойчивостью, а не степенной ${ }^{1)}$. Это препятствует конструированию достаточно богатого семейства таких решений с асимптотикой в виде степенных рядов [2]. С другой стороны, в рассматриваемой задаче сравнительно просто строится функция Ляпунова, и при анализе устойчивости можно обойтись без тех сложных конструкций, которые использовались в бездиссипативном случае [4].

Происхождение задачи. Системы типа (1) возникают при усреднении более сложных задач в теории колебаний [5], [6]. Переменные $\rho(t)$ и $\psi(t)$ описывают медленную эволюцию амплитуды (либо энергии) и сдвига фазы быстрых колебаний под действием малых осциллирующих возмущений. Положительный коэффициент $\gamma$ соответствует наличию диссипации, параметры $a, b$ связаны с амплитудой внешнего возмущения, слагаемое $\rho+\delta t+\delta_{0}$ характеризует рассогласование между собственной частотой и частотой возмущения. Специфика рассматриваемой нами задачи заложена в слагаемом $\rho$, которое отражает свойство неизохронности системы - зависимости собственной частоты от энергии. Единичный коэффициент при этом слагаемом обязан подходящей нормировке переменных $\rho$ и $t$.

В теории синхронизации неизохронность и неавтономность системы обычно игнорируется. В таком случае фазовое уравнение ${ }^{2)}$ имеет вид [7] (см. также книгу [1])

$$
\frac{d \psi}{d t}=\delta_{0}+b_{0} \sin \psi, \quad b_{0}, \delta_{0}=\text { const. }
$$

Легко понять, что общее решение $\psi(t)$ стабилизируется при $t \rightarrow \infty$ тогда и только тогда, когда существует устойчивое положение равновесия, т. е. при $\left|\delta_{0} / b_{0}\right|<1$. Это неравенство представляет собой условие синхронизации и интерпретируется как малое рассогласование частот.

В последнее время были обнаружены физические системы магнитодинамики [8], в которых неизохронность велика и должна учитываться в рассогласовании частот, как это указано в уравнениях (1). В автономном случае при $a=b=\delta=0$ такие уравнения исследовались в работе [9]. Было обнаружено сосуществование решений разного типа: как с синхронизацией, так и с проскальзыванием (неограниченным ростом) фазы при одних и тех же значениях параметров. Оказалось, что возможность синхронизации колебаний зависит не только от параметров системы, но и от начальных данных.

В настоящей работе рассматривается неизохронная система неавтономных уравнений (1). Неавтономность соответствует медленной деформации параметров внешней накачки в исходной системе (до усреднения). Интерес к такой задаче объясняется гипотезой о возможном расширении режима синхронизации в системе с медленной деформацией параметров.

Линейная зависимость от времени в коэффициентах уравнений берется из соображений реализуемости таких моделей в физических экспериментах. Задачи с более сложной зависимостью от $t$ и $\rho$, а также с общими периодическими функциями от $\psi$ могут представлять интерес в качестве математического упражнения.

1) В приложениях считается, что только устойчивые решения имеют отношение к описанию физически наблюдаемых явлений.

2)Это уравнение известно под названием уравнение Адлера. 
Результаты. Наличие каких-либо малых параметров в уравнениях не предполагается и не используется. В частности, не обсуждается возможность применения метода адиабатических приближений [6]. Вместо этого строится пара решений со степенной асимптотикой при $t \rightarrow \infty$. Затем доказывается, что одно из таких решений устойчиво по Ляпунову. Условия устойчивости выделяют область в пространстве параметров, которая ассоциируется с областью синхронизации.

\section{2. РЕШЕНИЯ СО СТЕПЕННОЙ АСИМПТОТИКОЙ}

Выделение решений, соответствующих синхронизации, в разных ситуациях может выполняться разными способами. Если система автономная, то ищутся неподвижные точки (положения равновесия); наличие устойчивого равновесия свидетельствует о синхронизации системы [9]. Для неавтономной системы в качестве аналога неподвижных точек следует рассматривать решения со степенной асимптотикой.

На первом этапе строятся асимптотические ряды

$$
\hat{\rho}(t)=\rho_{-1} t+\rho_{0}+\sum_{n=1}^{\infty} \rho_{n} t^{-n}, \quad \hat{\psi}(t)=\psi_{0}+\sum_{n=1}^{\infty} \psi_{n} t^{-n}, \quad t \rightarrow \infty .
$$

Их коэффициенты определяются из рекуррентной системы алгебраических уравнений, которая получается после подстановки этих рядов в (1) и приравнивания выражений при одинаковых степенях $t$. Например, на первых двух шагах получаются уравнения для $\rho_{-1}, \psi_{0}$ и $\rho_{0}, \psi_{1}$ :

$$
\begin{aligned}
-\gamma \rho_{-1}+a \cos \psi_{0} & =0, & \rho_{-1}-b \sin \psi_{0} & =-\delta . \\
-\gamma \rho_{0}-\psi_{1} a \sin \psi_{0} & =\rho_{-1}-a_{0} \cos \psi_{0}, & \rho_{0}-\psi_{1} b \cos \psi_{0} & =-\delta_{0}+b_{0} \sin \psi_{0} .
\end{aligned}
$$

Условия разрешимости таких уравнений являются необходимыми для существования решений типа (2). Эти условия индуцируют ограничения на параметры исходной задачи. Основное ограничение возникает при анализе нелинейной системы (3), которая редуцируется к тригонометрическому уравнению

$$
b \sin \psi_{0}-\frac{a}{\gamma} \cos \psi_{0}=\delta .
$$

Поскольку левая часть здесь представляет собой скалярное произведение вектора $(b,-a / \gamma)$ на единичный вектор $\left(\sin \psi_{0}, \cos \psi_{0}\right)$, для разрешимости уравнения относительно $\psi_{0}$ необходимо и достаточно выполнения условия $b^{2}+(a / \gamma)^{2} \geqslant \delta^{2}$. При этом существуют два корня $\psi_{0}=\psi_{0}^{ \pm}$(по модулю периода $2 \pi$ ). В ситуации общего положения, когда

$$
b^{2}+\left(\frac{a}{\gamma}\right)^{2}>\delta^{2}
$$

корни будут простыми. При таком условии линейные системы алгебраических уравнений типа (4), которые встречаются на последующих шагах, однозначно разрешимы, и из них находятся две серии коэффициентов

$$
\rho_{n}=\rho_{n}^{ \pm}, \quad \psi_{n+1}=\psi_{n+1}^{ \pm}, \quad n=0,1, \ldots .
$$


Таким способом строятся два асимптотических решения в виде рядов (2). Ряды, конечно, расходятся. Тем не менее существуют точные решения, гладкие на полуоси, например при $t \geqslant 0$, для которых эти ряды представляют собой асимптотику при $t \rightarrow \infty$ [10], так что имеет место следующее утверждение.

Теорема 1. Пусть выполнено условие (6). Тогда система уравнений (1) имеет пару решений со степенной асимптотикой (2). Каждая из двух возможных констант $\psi_{0}$, на которой стабилизируется фазовая функиия $\psi(t)$, удовлетворяет соотношению (5).

Отметим, что неравенство (6) напоминает известное условие синхронизации, которое возникает в автономной системе из анализа уравнения Адлера [1], [8], [9]. В рассматриваемом нами неавтономном случае условие (6) имеет тот же смысл: рассогласование частот должно быть не слишком велико по сравнению с амплитудой накачки. Это ограничение достаточно для существования пары решений типа (2); оно почти необходимо, как показывает следующее утверждение.

ТЕОРема 2. Если параметры системы (1) удовлетворяют неравенству

$$
b^{2}+\left(\frac{a}{\gamma}\right)^{2}<\delta^{2}
$$

то не существует решений с фазой $\psi(t)$, ограниченной $n р и t \rightarrow \infty$.

ДокАЗАТЕЛьство. Используем тождество

$$
\frac{d}{d t}\left(\frac{\rho}{\gamma}+\psi\right)=t\left(\delta+\frac{a}{\gamma} \cos \psi-b \sin \psi\right)+\left(\delta_{0}+\frac{a_{0}}{\gamma} \cos \psi-b_{0} \sin \psi\right),
$$

справедливое на решении системы. Считая параметр $\delta>0$ положительным (без ограничения общности), отсюда получаем оценку производной

$$
\frac{d}{d t}\left(\frac{\rho}{\gamma}+\psi\right) \geqslant m t-m_{0}, \quad m, m_{0}=\mathrm{const}, \quad m=\delta-\sqrt{b^{2}+\frac{a^{2}}{\gamma^{2}}}>0, \quad m_{0}>0 .
$$

После интегрирования этого неравенства получается соотношение

$$
\frac{\rho(t)}{\gamma}+\psi(t) \geqslant \frac{m t^{2}}{2}-m_{0} t+\text { const, } \quad t>0
$$

Из него следует, что если фазовая функция $\psi(t)$ ограничена, то график амплитуды $\rho(t)$ при $t \rightarrow \infty$ расположен над быстро растущей кривой $\rho=\gamma\left(m t^{2} / 2-m_{0} t+\right.$ const $)$. Однако функция $\rho(t)$ обязана монотонно убывать при $\rho>\left(a t+a_{0}\right) / \gamma$, поскольку ее производная отрицательна в силу первого уравнения из (1). Это противоречие показывает, что предположение об ограниченности $\psi(t)$ неверно. Теорема доказана.

С явлением синхронизации связываются лишь устойчивые решения, а такое свойство не гарантируется условием (6). Вопрос устойчивости одного из построенных выше решений оказывается более сложным по сравнению с автономным случаем. Этому посвящена оставшаяся часть настоящей работы. 


\section{3. АСИМПТОТИКА ВКБ ДЛЯ ОСТАТКА}

Пусть пара функций $\hat{\rho}(t), \hat{\psi}(t)$ представляет частное решение уравнений $(1)$, которое имеет асимптотическое разложение (2) с одним из двух возможных корней $\psi_{0}$. Для обсуждения вопроса устойчивости по Ляпунову (устойчивости относительно возмущения начальных данных) выделим эти функции из общего решения путем замены $\rho(t)=\hat{\rho}(t)+R(t), \psi(t)=\hat{\psi}(t)+\Psi(t)$. После этого проблема сводится к вопросу устойчивости положения равновесия $R \equiv 0, \Psi \equiv 0$ для системы

$$
\begin{aligned}
& \frac{d R}{d t}=-\gamma R+\left(a t+a_{0}\right)[\cos (\hat{\psi}+\Psi)-\cos \hat{\psi}], \\
& \frac{d \Psi}{d t}=R-\left(b t+b_{0}\right)[\sin (\hat{\psi}+\Psi)-\sin \hat{\psi}] .
\end{aligned}
$$

Один из возможных способов анализа устойчивости основан на изучении уравнений, линеаризованных в точке равновесия. В данном случае линеаризованная система имеет вид

$$
\frac{d}{d t}\left(\begin{array}{l}
R \\
\Psi
\end{array}\right)=\left(\begin{array}{cc}
-\gamma & -A(t) \\
1 & -B(t)
\end{array}\right)\left(\begin{array}{l}
R \\
\Psi
\end{array}\right)
$$

Здесь $A=\left(a t+a_{0}\right) \sin \hat{\psi}, B=\left(b t+b_{0}\right) \cos \hat{\psi}$. В этой системе для пары линейно независимых решений можно выписать асимптотику на бесконечности в виде формул, которые иногда называются приближениями ВКБ [11]:

$$
\left(\begin{array}{l}
R \\
\Psi
\end{array}\right)=\exp \left\{\int_{0}^{t} \lambda_{ \pm}(t) d t\right\}\left(\begin{array}{l}
R_{ \pm}(t) \\
\Psi_{ \pm}(t)
\end{array}\right)[1+o(1)], \quad t \rightarrow \infty .
$$

Показатель экспоненты здесь определяется через собственные значения матрицы системы (9)

$$
\lambda_{ \pm}(t)=-\frac{1}{2}(\gamma+B) \pm \sqrt{\frac{1}{4}(\gamma-B)^{2}-A},
$$

а $R_{ \pm}, \Psi_{ \pm}-$компоненты соответствующего собственного вектора. Эти функции имеют степенные асимптотики на бесконечности. Поэтому экспоненциальная устойчивость или неустойчивость равновесия в линейной системе определяется главным членом асимптотики собственных значений

$$
\begin{aligned}
\lambda_{+}(t) & =-\lambda_{0}+O\left(t^{-1}\right), & \lambda_{-}(t) & =-\lambda_{1} t+O(1), \quad t \rightarrow \infty, \\
\lambda_{0} & =\gamma+\frac{a}{b} \operatorname{tg} \psi_{0}, & \lambda_{1} & =b \cos \psi_{0} .
\end{aligned}
$$

В частности, условия $b \cos \psi_{0}>0, b \cos \psi_{0}+(a / \gamma) \sin \psi_{0}>0$ необходимы и достаточны, чтобы оба собственных значения были отрицательны в окрестности бесконечности. При таких условиях линеаризованные уравнения (9) имеют фундаментальную систему решений, стремящихся к нулю на бесконечности, и, следовательно, равновесие асимптотически устойчиво.

Полученный результат можно использовать, чтобы доказать устойчивость равновесия для исходной нелинейной системы (8). Известные способы доказательства опираются на получение оценки для остатка асимптотики с использованием функции типа Ляпунова. Из этих соображений представляется более естественным проводить анализ устойчивости непосредственно для нелинейной системы (8), применяя второй метод Ляпунова. Линеаризованная система используется при конструировании функции Ляпунова. 


\section{4. ФУНКЦИЯ ЛЯПУНОВА}

Конструкция функции Ляпунова основана на приведении к диагональной форме главной (при $t \rightarrow \infty$ ) части линеаризованной системы (9). Для этого используются собственные значения $\lambda_{ \pm}(t)$ и матрица, составленная из правых собственных векторов:

$$
\mathcal{M}(t)=\left(\begin{array}{ll}
R_{+} & R_{-} \\
\Psi_{+} & \Psi_{-}
\end{array}\right)
$$

где

$$
\begin{array}{ll}
R_{+}=\frac{A}{A-\left(\gamma+\lambda_{+}\right)^{2}}, & R_{-}=\frac{A\left(\gamma+\lambda_{-}\right)}{A-\left(\gamma+\lambda_{-}\right)^{2}}, \\
\Psi_{+}=\frac{\gamma+\lambda_{+}}{\left(\gamma+\lambda_{+}\right)^{2}-A}, & \Psi_{-}=\frac{\left(\gamma+\lambda_{-}\right)^{2}}{\left(\gamma+\lambda_{-}\right)^{2}-A} .
\end{array}
$$

Обратная матрица имеет простой вид,

$$
\mathcal{M}^{-1}(t)=\left(\begin{array}{cc}
1 & \gamma+\lambda_{+} \\
\left(\gamma+\lambda_{-}\right)^{-1} & 1
\end{array}\right)
$$

При такой нормировке собственных векторов элементы обеих матриц стабилизируются на бесконечности:

$$
\mathcal{M}(t)=\text { const }+O\left(t^{-1}\right), \quad \mathcal{M}^{-1}(t)=\text { const }+O\left(t^{-1}\right), \quad t \rightarrow \infty .
$$

После замены функций по формуле

$$
\left(\begin{array}{l}
R \\
\Psi
\end{array}\right)=\mathcal{M}(t)\left(\begin{array}{l}
x \\
y
\end{array}\right)
$$

система линейных уравнений приводится к виду

$$
\frac{d}{d t}\left(\begin{array}{l}
x \\
y
\end{array}\right)=\Lambda(t)\left(\begin{array}{l}
x \\
y
\end{array}\right)-B(t)\left(\begin{array}{l}
x \\
y
\end{array}\right)
$$

Здесь $\Lambda(t)=\operatorname{diag}\left\{\lambda_{+}(t), \lambda_{-}(t)\right\}$ - диагональная матрица с элементами $\lambda_{ \pm}$. Дополнительная матрица, определяемая по формуле

$$
B(t)=\mathcal{M}^{-1}(t) \mathcal{M}^{\prime}(t)=O\left(t^{-1}\right), \quad t \rightarrow \infty,
$$

стремится к нулю на бесконечности из-за наличия производной $\mathcal{M}^{\prime}(t)$ и стабилизации матриц $\mathcal{M}^{-1}(t), \mathcal{M}(t)$. Отметим, что именно это свойство обеспечивает справедливость асимптотической формулы (10) (см. книгу [11]).

С другой стороны, указанное свойство позволяет построить функцию Ляпунова в виде квадратичной формы $U(x, y)=\left(x^{2}+y^{2}\right) / 2$. Более того, такая функция подходит для исходной нелинейной системы, которая в новых переменных имеет вид

$$
\frac{d}{d t}\left(\begin{array}{l}
x \\
y
\end{array}\right)=\Lambda(t)\left(\begin{array}{l}
x \\
y
\end{array}\right)-B(t)\left(\begin{array}{l}
x \\
y
\end{array}\right)+\left(\begin{array}{l}
F_{1}(x, y, t) \\
F_{2}(x, y, t)
\end{array}\right) .
$$


Здесь вектор нелинейной части определяется формулой

$$
\left(\begin{array}{l}
F_{1} \\
F_{2}
\end{array}\right)=\mathcal{M}^{-1}(t)\left(\begin{array}{c}
\left(a t+a_{0}\right)[\cos (\hat{\psi}+\Psi)-\cos \hat{\psi}+\Psi \sin \hat{\psi}] \\
\left(b t+b_{0}\right)[\sin (\hat{\psi}+\Psi)-\sin \hat{\psi}-\Psi \cos \hat{\psi}]
\end{array}\right) .
$$

Заметим, что нелинейная часть в (15) зависит от одной компоненты $\Psi$ исходного искомого вектора. Эта компонента связана с новыми переменными соотношением

$$
\Psi=\Psi_{+} x+\Psi_{-} y \equiv \frac{\gamma+\lambda_{+}}{\left(\gamma+\lambda_{+}\right)^{2}-A} x+\frac{\left(\gamma+\lambda_{-}\right)^{2}}{\left(\gamma+\lambda_{-}\right)^{2}-A} y .
$$

В силу асимптотики собственных значений (12) и выражения для $A=\left(a t+a_{0}\right) \sin \hat{\psi}$ из последней формулы получается оценка

$$
\Psi^{2} \leqslant M\left[\left(\frac{x}{t}\right)^{2}+y^{2}\right]
$$

с некоторой константой $M>0$, равномерная для всех достаточно больших $t \geqslant t_{0}$ и для всех $x, y$ из окрестности $x^{2}+y^{2}<1$ положения равновесия.

Поскольку в окрестности положения равновесия нелинейные добавки в уравнениях оцениваются через $\Psi^{2}$,

$$
|\cos (\hat{\psi}+\Psi)-\cos \hat{\psi}+\Psi \sin \hat{\psi}| \leqslant M \Psi^{2}, \quad|\sin (\hat{\psi}+\Psi)-\sin \hat{\psi}-\Psi \cos \hat{\psi}| \leqslant M \Psi^{2},
$$

ввиду ограниченности матрицы $\mathcal{M}^{-1}(t)$ компоненты $F_{1}, F_{2}$ оцениваются через квадратичные слагаемые в переменных $x, y$ :

$$
\left|F_{1}(x, y, t)\right|,\left|F_{2}(x, y, t)\right| \leqslant M\left[\frac{x^{2}}{t}+t y^{2}\right] .
$$

\section{5. УСТОЙЧИВОСТЬ СТЕПЕННОГО РЕШЕНИЯ}

Докажем следующее утверждение.

Теорема 3. Пусть решение системы (1) имеет степенную асимптотику (2), так что главный член фазы $\psi_{0}$ удовлетворяет уравнению (5). Если исходные параметры $a, b, \gamma, \delta$ удовлетворяют неравенствам

$$
b \cos \psi_{0}>0, \quad b \cos \psi_{0}+\frac{a}{\gamma} \sin \psi_{0}>0,
$$

то решение с асимптотикой (2) экспоненциально устойчиво по Ляпунову.

ДокАзАТЕЛьство воспроизводит известное доказательство теоремы Ляпунова (см., например, монографии [12], [13]). В окрестности бесконечности $t>t_{0}$ система (8) эквивалентна уравнениям (15). В качестве функции Ляпунова возьмем квадратичную форму $U(x, y)=\left(x^{2}+y^{2}\right) / 2$. Выпишем полную производную вдоль траектории системы (15) через скалярные произведения векторов:

$$
\begin{aligned}
\frac{d U}{d t} & =(x, y) \frac{d}{d t}\left(\begin{array}{l}
x \\
y
\end{array}\right)= \\
& =\lambda_{+}(t) x^{2}+\lambda_{-}(t) y^{2}+(x, y) B(t)\left(\begin{array}{l}
x \\
y
\end{array}\right)+(x, y) \cdot\left(\begin{array}{l}
F_{1}(x, y, t) \\
F_{2}(x, y, t)
\end{array}\right) .
\end{aligned}
$$


Выделим здесь главные члены асимптотики при $t \rightarrow \infty$ в окрестности положения равновесия, используя формулы (12) и оценки (14), (16):

$$
\frac{d U}{d t}=-\lambda_{0} x^{2}-\left[\lambda_{1} t+O(1)\right] y^{2}+O\left(t^{-1}\right)\left(x^{2}+y^{2}\right)+\left[O\left(|x|^{3}\right)+t O\left(|y|^{3}\right)\right] .
$$

Асимптотическое соотношение можно заменить неравенством, оценив сверху все остаточные члены. Имеем

$$
\frac{d U}{d t} \leqslant-\lambda_{0} x^{2}-\left(\lambda_{1} t-M\right) y^{2}+M t^{-1}\left(x^{2}+y^{2}\right)+M(|x|+|y|)\left(x^{2}+t y^{2}\right),
$$

где $M=$ const. Отсюда видно, что знак производной определяется знаком констант $-\lambda_{0}$ и $-\lambda_{1}$ равномерно для всех достаточно больших $t>t_{0}$ в достаточно малой окрестности $x^{2}+y^{2}<\Delta$ положения равновесия. В частности, при условиях (17) константы $-\lambda_{0}<0$ и $-\lambda_{1}<0$, поэтому производная будет отрицательна. $\mathrm{B}$ таком случае асимптотическая устойчивость равновесия в системе (8) при $t>t_{0}$ следует из известных результатов [13].

Более того, для производной получается грубая оценка

$$
\frac{d U}{d t} \leqslant-m U
$$

с положительной константой $m>0$, равномерная в окрестности положения равновесия и при $t>t_{0}$. Отсюда следует, что равновесие экспоненциально устойчиво, поскольку вдоль траектории

$$
\frac{1}{2}\left(x^{2}+y^{2}\right) \equiv U(x, y) \leqslant \text { const } \cdot e^{-m t} .
$$

На конечном промежутке $0 \leqslant t \leqslant t_{0}$ устойчивость следует из теоремы о непрерывной зависимости решения от начальных данных. Заметим, что глобальная разрешимость задачи Коши для уравнений (8) и устойчивость равновесия (на промежутке $\left.0 \leqslant t \leqslant t_{0}\right)$ обеспечивается простой структурой правых частей (см. книгу [14]). Теорема доказана.

ЗАмЕчАниЕ 1. Утверждение об устойчивости выглядит более слабым по сравнению с асимптотической формулой типа (10). Но зато его можно использовать при постоянно действующих возмущениях [13], [15], когда нельзя выписать никакие асимптотические приближения.

При анализе теоремы может возникнуть вопрос, выполняются ли когда-либо условия, которые задаются в неявной форме (17) через корень уравнения (5). Этот вопрос легко решается, если в качестве независимых параметров использовать другой набор, включающий $\psi_{0}$, например $a, b, \gamma, \psi_{0}$. При этом исходная задача должна ставиться в более общей форме: требуется найти ограничения на параметры системы $a, b, \gamma, \delta$, при которых существует устойчивое решение с асимптотикой (2) при значении главного члена фазы, равном $\psi_{0}$. В таком подходе фигурирует пятерка параметров $a, b, \gamma, \delta, \psi_{0}$, которая в силу того, что мы рассматриваем решение $(2)$, связана соотношением (5). Таким образом, один из параметров надо считать зависимым. Например, $\delta$ можно определять равенством (5). Тогда условия (17) описывают 
область устойчивости в пространстве параметров $a, b, \gamma, \psi_{0}$. Легко видеть, что при заданных значениях $a, b, \gamma$ всегда имеется широкая область доступных значений $\psi_{0}$.

Заметим, что из двух различных корней уравнения (5) лишь один удовлетворяет второму условию в (17). Поэтому теорема об устойчивости применима к одному из двух степенных решений (2). $\mathrm{K}$ другому решению иногда применима теорема Ляпунова о неустойчивости.

Теорема 4. Пусть решение системъ (1) имеет степенную асимптотику (2), так что главный член $\psi_{0}$ удовлетворяет уравнению (5). Если исходные параметры $a, b, \gamma, \delta$ удовлетворяют неравенствам

$$
b \cos \psi_{0}<0, \quad b \cos \psi_{0}+\frac{a}{\gamma} \sin \psi_{0}<0,
$$

то решение (2) неустойчиво по Ляпунову.

ДокАЗАТЕЛьство. В рассматриваемом случае остаточные члены в выражении для полной производной оцениваются снизу:

$$
\frac{d U}{d t} \geqslant-\lambda_{0} x^{2}-\left(\lambda_{1} t-M\right) y^{2}-M t^{-1}\left(x^{2}+y^{2}\right)-M(|x|+|y|)\left(x^{2}+t y^{2}\right) .
$$

Поскольку при условиях (18) числа $\lambda_{0}, \lambda_{1}$ отрицательны, при больших $t>t_{0}$ производная оказывается положительной равномерно в малой окрестности $x^{2}+y^{2}<\Delta$, и, следовательно, равновесие неустойчиво. Теорема доказана.

ЗАмЕчАниЕ 2. Если $\delta \neq 0$, то имеется область параметров, в которой нарушаются условия обеих теорем. Эта область определяется неравенством

$$
\left(b \cos \psi_{0}+\frac{a}{\gamma} \sin \psi_{0}\right) b \cos \psi_{0}<0 .
$$

При таких значениях параметров теоремы Ляпунова не годятся для анализа устойчивости. С другой стороны, линеаризованное приближение свидетельствует о неустойчивости, поскольку собственные значения $\lambda_{ \pm}(t)$ имеют разный знак при $t \rightarrow \infty$. Однако приложение этого результата к нелинейной системе остается необоснованным.

\section{6. ЗАКЛЮЧЕНИЕ}

Исследована задача о выделении пятерки параметров $a, b, \gamma, \delta, \psi_{0}$, при которых уравнения (1) имеют устойчивые решения с компонентой $\psi(t)$, стабилизирующейся на бесконечности на значении $\psi_{0}$. Такие решения ассоциируются с явлением синхронизации (захвата фазы).

При условии $b^{2}+(a / \gamma)^{2}>\delta^{2}$ гарантируется существование пары решений $(2)$, у которых фаза стабилизируется при $t \rightarrow \infty$. Предельное значение $\psi_{0}$ обязано удовлетворять соотношению (5),

$$
\delta=b \sin \psi_{0}-\frac{a}{\gamma} \cos \psi_{0},
$$

так что независимыми являются четыре параметра. Одно из решений с асимптотикой (2) устойчиво по Ляпунову при условиях (17). 
Отметим, что, помимо решений с синхронизацией, у системы (1) существуют решения с проскальзыванием фазы, когда

$$
\psi(t)=s_{2} t^{2}+s_{1} t+O(1), \quad t \rightarrow \infty,
$$

где $s_{1,2}$ - отличные от нуля константы. Необходимым и достаточным условием для этого является большое рассогласование частот: $\delta^{2}>b^{2}$. Из сравнения этого неравенства с условием (6) следует, что при $\delta \neq 0$ имеется значительная область параметров

$$
b^{2}+\left(\frac{a}{\gamma}\right)^{2}>\delta^{2}>b^{2}
$$

при которых существуют решения двух типов: как с захватом, так и с проскальзыванием фазы. Подобный эффект был ранее обнаружен в автономной системе [9]; впрочем, такая ситуация является обычной во многих задачах об авторезонансе [2]. Более детальный анализ проскальзывания фазы в задаче (1) выходит за рамки настоящей статьи.

При $\delta=0$ и $b \neq 0$ проскальзывания фазы не бывает. Корни $\psi_{0}=\psi_{0}^{ \pm}$алгебраического уравнения (5) определяются соотношениями

$$
\sin \psi_{0}^{ \pm}= \pm \frac{1}{\sqrt{(a / \gamma)^{2}+b^{2}}} \frac{a}{\gamma}, \quad \cos \psi_{0}^{ \pm}= \pm \frac{1}{\sqrt{(a / \gamma)^{2}+b^{2}}} b .
$$

Легко проверить, что для соответствующих решений (2) выполняются условия либо теоремы 3 (в случае знака плюс), либо теоремы 4 (в случае знака минус).

Для решений с ограниченной фазой $\psi(t)$ амплитуда $\rho(t)$ в общем случае неограничена при $t \rightarrow \infty$ вследствие линейного роста коэффициентов уравнений (1). Из-за этого может создаться впечатление, что применительно к задачам синхронизации такие решения не имеют подходящей физической интерпретации. Однако надо помнить, что уравнения (1) применимы для описания явления синхронизации лишь на начальном этапе захвата в резонанс. Математическая модель в форме уравнений (1) пригодна на не слишком больших временах: при $0<t \ll \varepsilon^{-1}$. Здесь $\varepsilon-$ малый параметр, характеризующий амплитуду накачки в исходной задаче (до усреднения). Такое ограничение соответствуют применимости приближения по малой амплитуде $\varepsilon|\rho(t)| \ll 1$. В задаче о синхронизации неограниченный рост амплитуды свидетельствует о медленном уходе траектории от предельного цикла под действием малой медленно растущей накачки. Важно, что такой процесс происходит в режиме синхронизации, т. е. при стабилизирующейся расстройке фаз.

Наличие функции Ляпунова и свойство асимптотической устойчивости обеспечивает устойчивость рассматриваемого степенного решения относительно постоянно действующих возмущений [15]. Класс подходящих возмущений выделяется требованием их малости вдоль решений с растущей амплитудой $\rho(t)=O(t)$ при $t \rightarrow \infty$; подобные результаты содержатся, например в работах [4], [16].

Кроме того, наличие функции Ляпунова обеспечивает априорную оценку для решения системы (1). Это дает возможность доказать теорему существования решения с заданной асимптотикой обычным способом [14], не обращаясь к результатам труднопроверяемой работы [10].

Благодарности. Работа выполнена при поддержке РНФ (грант № 14-11-00078). 


\section{Список литературы}

[1] А. Пиковский, М. Розенблюм, Ю. Куртс, Синхронизация. Фундаментальное нелинейное явление, Техносфера, М., 2003.

[2] Л. А. Калякин, УМН, 63:5(383) (2008), 3-72.

[3] Л.А. Калякин, М. А. Шамсутдинов, ТМФ, 160:1 (2009), 102-111.

[4] Л. А. Калякин, О. А. Султанов, Дифференциалъные уравнения, 49:3 (2013), 279-293.

[5] Н.Н. Боголюбов, Ю.А. Митропольский, Асимптотические методы в теории нелинейных колебаний, Наука, М., 1974.

[6] В. И. Арнольд, В. В. Козлов, А. И. Нейштадт, "Математические аспекты классической и небесной механики", Динамические системы-3, Итоги науки и техники. Современные проблемы математики. Фундаментальные направления, 3, ВИНИТИ, М., 1985, 5-290.

[7] R. Adler, Proc. IRE, 34:6 (1946), 351-357.

[8] A. Slavin, V. Tiberkevich, IEEE Transaction on Magnetics, 45:4 (2009), 1875-1918.

[9] Л. А. Калякин, Ж. вычисл. матем. и матем. физ., 50:8 (2010), 1408-1419.

[10] А.Н. Кузнецов, Функи. анализ и его прил., 6:2 (1972), 41-51.

[11] М. В. Федорюк, Асимптотические методы для линейных обыкновенных дифференииальных уравнений, Наука, М., 1983.

[12] М. А. Ляпунов, Общая задача об устойчивости движения, Гостехиздат, М.-Л., 1950.

[13] Н.Н. Красовский, Некоторые задачи теории устойчивости движения, Физматгиз, M., 1959.

[14] В. В. Немыцкий, В. В. Степанов, Качественная теория дифференциалъных уравнений, Едиториал, М., 2004.

[15] И. Г. Малкин, Теория устойчивости движения, Гостехиздат, М.-Л., 1952.

[16] О. А. Султанов, Тр. ИММ УрО РАН, 19:3 (2013), 274-283. 